\title{
A diagnosis of isopycnal mixing by mesoscale eddies
}

\author{
Carsten Eden and Richard J. Greatbatch
}

IFM-GEOMAR, Kiel, Germany

Manuscript submitted in revised form to Ocean Modelling, Dec. 2008

Corresponding author address:

Carsten Eden

IFM-GEOMAR,

FB I, Ocean circulation and climate dynamics

Düsternbrooker Weg 20

24105 Kiel, Germany

email: ceden@ifm-geomar.de 


\section{Abstract}

Combining the buoyancy and tracer budget in the generalised Temporal Residual Mean (TRM-G) framework of Eden et al. (2007a), we show that within the small slope approximation and weakly diabatic situation, the isopycnal diffusivity is related to the difference of the streamfunctions of the eddy-induced velocities of tracer and buoyancy divided by the angle between the (negative) slopes of isopycnals and the isolines of the tracer. Using this result tracer simulations of a realistic mesoscaleeddy-permitting model of the North Atlantic coupled to a biogeochemical model are diagnosed in terms of zonal $\left(K_{I}^{(x)}\right)$ and meridional $\left(K_{I}^{(y)}\right)$ isopycnal diffusivities relevant for non-eddy-permitting ocean models.

We find for tracers having different interior sources and surface forcing and therefore different lateral and vertical mean gradients, values of $K_{I}^{(x)}$ and $K_{I}^{(y)}$ with similar magnitudes and lateral and vertical structure. In general, isopycnal diffusivities lie within the expected range between 0 and 5000 $\mathrm{m}^{2} / \mathrm{s}$ but we also find a strong anisotropy with $K_{I}^{(x)}$ much larger than $K_{I}^{(y)}$ over large regions of the North Atlantic. Both $K_{I}^{(x)}$ and $K_{I}^{(y)}$ are larger within and above the thermocline but decay almost to zero below. Our results also support the common practise of the use of identical isopycnal and thickness diffusivity for any tracer in ocean models.

\section{Introduction}

Ocean general circulation models (OGCMs) aim to simulate the large-scale oceanic circulation and its buoyancy and tracer distributions which are characterised by lateral changes over scales as large as the ocean basins. When in OGCMs the energetic mesoscale fluctuations on the much smaller scales of several to about $100 \mathrm{~km}$ remain unresolved, their effects have to be parameterised. An important application of such parameterised OGCMs is their use as components of climate models to predict for instance the uptake of carbon dioxide from the atmosphere in future global climate change (Houghton et al., 2001). The simulation of the realistic ventilation of the interior ocean is of particular importance for the oceanic carbon draw-down. Aside from ventilation of the interior ocean by the large scale flow field, e.g. by Ekman pumping (Luyten et al., 1983), another mechanism is the mixing of tracers along mean isopycnals into the interior by mesoscale eddy activity. Isopycnal mixing and its parameterisation in OGCMs is the focus of the present study. 
Our approach is to consider at the same time the budgets for mean buoyancy and a mean tracer in the Transformed Eulerian Mean framework (TEM) of Andrews et al. (1987) or, more specifically in the generalisation of TEM (TRM-G) of Eden et al. (2007a). In the TEM (TRM-G) framework the effect of mesoscale fluctuations on the mean buoyancy budget is split into an (apparent) advective and a diffusive effect, while a rotational part with no effect on the mean buoyancy is separated out. The diffusive effect is expressed by a turbulent diffusivity mixing the mean buoyancy across isolines of mean buoyancy (isopycnals) and is often small and therefore often neglected, while the advective effect is given by a streamfunction for an eddy-induced advection velocity which adds to the mean velocity in the mean budget. The latter is often parameterised in ocean models by the closure of Gent and McWilliams (1990).

Although the TEM framework was originally suggested to be applied for the buoyancy budget, it can also be used for any tracer. For each individual mean tracer, however, different eddy-induced velocities and different turbulent diffusivities will in general show up. The TRM-G framework of Eden et al. (2007a) relates the turbulent diffusivity to the structure of the mean field and the dissipation or other sources and sinks (such as micro-scale diffusion, absorption of solar radiation, remineralisation of organic matter, etc) of the respective tracer, raising therefore the possibility of different turbulent diffusivities and consequently of different eddy-induced velocities for tracers with different sources (Greatbatch, 2001). On the other hand, it is certainly of practical benefit for an ocean model to use identical eddy-induced velocities $\left(\boldsymbol{u}^{*}\right)$ for each tracer. The remainder of the mesoscale eddy effect in the mean tracer budget is then usually interpreted as diffusion along mean isopycnals (Redi, 1982). In current OGCMs, the magnitude (and direction) of the diffusive flux along isopycnals is given by the so-called isopycnal diffusivity (tensor), usually taken identical for any tracer and also identical to the lateral (thickness) diffusivity used in the Gent and McWilliams (1990) parameterisation. As a consequence for practical use in a non-eddy-permitting ocean model, there is only the need to find a parameterisation for a single $\boldsymbol{u}^{*}$, i.e. the one for buoyancy, and, eventually, a parameterisation for the isopycnal (and diapycnal) diffusivity. We investigate the consequences of this practical approach in the TRM-G framework and assume identical eddy-induced velocities $\boldsymbol{u}^{*}$ for buoyancy and tracers and interpret the differences in $\boldsymbol{u}^{*}$ (and diffusivity) for tracer and buoyancy as isopycnal (and diapycnal) diffusion.

Before developing a parameterisation it is useful to consider observational estimates of the lateral and vertical structure of isopycnal diffusivities. Since interior oceanic observations of mesoscale fluctuations are in general rather sparse such that the significance of a respective analysis gets low, it is current practise to rely on pseudo observations of mesoscale-eddy-permitting model simulations, e. g. Rix and Willebrand (1996); Jochum (1997); Bryan et al. (1999); Treguier (1999); Nakamura and 
Chao (2000); Roberts and Marshall (2000); Drijfhout and Hazeleger (2001); Peterson and Greatbatch (2001); Solovev et al. (2002). In this study we diagnose the isopycnal diffusivity from the results of a realistic mesoscale-eddy-permitting model of the North Atlantic. The model is coupled to a standard biogeochemical model (Eden and Oschlies, 2006) providing realistic prognostic budgets for nitrate, oxygen and dissolved inorganic carbon. In addition, we use temperature and salinity to obtain five independent long-term averages of the eddy tracer fluxes in the model. The eddy buoyancy fluxes from the same model were used by Eden et al. (2007b) to diagnose the thickness diffusivity appropriate for the Gent and McWilliams (1990) parameterisation. It was found by Eden et al. (2007b) that a scalar thickness diffusivity is not sufficient to represent the eddy buoyancy fluxes, but a tensor is needed having two independent components related to the strongly anisotropic lateral mixing of buoyancy. We also find in this study based on the model diagnosis the need for anisotropic lateral isopycnal diffusivity.

In the following sections, we will discuss the general relation between buoyancy and individual tracers with respect to eddy-driven advection, isopycnal and diapycnal mixing within the TRM-G framework for the two-dimensional (section 2) and the three-dimensional case (section 3). We will estimate in section 4 the along isopycnal mixing in terms of an isopycnal diffusivity tensor from results of an mesoscale-eddy-permitting ocean model of the Atlantic Ocean coupled to a simple nitrate-based ecosystem/biogeochemical model, while in section 5 the results are summarised and discussed.

\section{Isopycnal diffusivity in the TRM-G framework}

Consider the budgets for buoyancy $b$ and a tracer concentration $T$ in the Boussinesq approximation. We decompose buoyancy, tracer and velocity into zonal mean and deviation (denoted by primes) and take the zonal average (denoted by an overbar) of the buoyancy and tracer budget. We discuss the three-dimensional case of a temporal mean in section 3. The zonal mean buoyancy and tracer budgets are given by

$$
\begin{aligned}
\bar{b}_{t}+\overline{\boldsymbol{u}} \cdot \nabla \bar{b}+\nabla \cdot \overline{\boldsymbol{u}^{\prime} b^{\prime}} & =\bar{Q}_{b} \\
\bar{T}_{t}+\overline{\boldsymbol{u}} \cdot \nabla \bar{T}+\nabla \cdot \overline{\boldsymbol{u}^{\prime} T^{\prime}} & =\bar{Q}_{T}
\end{aligned}
$$

Interior small-scale processes like micro-scale diffusion and other sources and sinks of buoyancy or tracer are denoted by $Q_{b}$ and $Q_{T}$ respectively. Note that in this section, the $\nabla$-operator and the velocity vector are two-dimensional in the meridional-vertical plane, due to the zonal averaging. Following the TRM-G framework, we decompose the buoyancy $\left(\overline{\boldsymbol{u}^{\prime} b^{\prime}}\right)$ and tracer eddy flux $\left(\overline{\boldsymbol{u}^{\prime} T^{\prime}}\right)$ into rotational 
fluxes and components along and across isolines of mean buoyancy and tracer, which yields

$$
\begin{aligned}
\bar{b}_{t}+\left(\overline{\boldsymbol{u}}-\nabla B_{b}\right) \cdot \nabla \bar{b} & =\nabla \cdot K_{b} \nabla \bar{b}+\bar{Q}_{b} \\
\bar{T}_{t}+\left(\overline{\boldsymbol{u}}-\nabla B_{T}\right) \cdot \nabla \bar{T} & =\nabla \cdot K_{T} \nabla \bar{T}+\bar{Q}_{T}
\end{aligned}
$$

The operator $\nabla$ is given by $\nabla=\left(-\frac{\partial}{\partial z}, \frac{\partial}{\partial y}\right)^{T}$, i. e. a shorthand ${ }^{1}$ for $\boldsymbol{e}_{1} \times \nabla$. The turbulent diffusivities $K_{b}$ and $K_{T}$ are given by

$$
K_{b}=-|\nabla \bar{b}|^{-2}\left(\overline{\boldsymbol{u}^{\prime} b^{\prime}}-\nabla \theta_{b}\right) \cdot \nabla \bar{b} \quad \text { and } \quad K_{T}=-|\nabla \bar{T}|^{-2}\left(\overline{\boldsymbol{u}^{\prime} T^{\prime}}-\nabla \theta_{T}\right) \cdot \nabla \bar{T}
$$

Note that $K_{b}$ is related to the cross-isopycnal (diapycnal) eddy flux and thus denotes a diapycnal diffusivity. The streamfunctions for eddy-induced velocities are given by

$$
B_{b}=|\nabla \bar{b}|^{-2}\left(\overline{\boldsymbol{u}^{\prime} b^{\prime}}-\underset{\neg}{\nabla} \theta_{b}\right) \cdot \underset{\neg}{\nabla} \quad \text { and } \quad B_{T}=|\nabla \bar{T}|^{-2}\left(\overline{\boldsymbol{u}^{\prime} T^{\prime}}-\underset{\neg}{\nabla} \theta_{T}\right) \cdot \underset{\neg}{\bar{T}}
$$

We have accounted for rotational components in the eddy buoyancy and tracer fluxes, $\underset{\neg}{\nabla} \theta_{b}$ and $\underset{\neg}{\nabla} \theta_{T}$, which are subtracted from the raw fluxes and for which a physically meaningful definition is given by the TRM-G framework of Eden et al. (2007a). The rotational components drop out taking the divergence and thus do not affect the mean tracer budget, but do affect the definition of $K_{b}, K_{T}, B_{b}$ and $B_{T}$ as discussed in Eden et al. (2007a). Note that in Eq. (3) and Eq. (4) there are two different eddy-induced velocities for buoyancy and the tracer. Note also that the representation in Eq. (5) and in Eq. (6) is valid only for $|\nabla \bar{b}| \neq 0$, such that we cannot consider situations with nonzero eddy buoyancy (tracer) fluxes in the presence of vanishing gradients of mean buoyancy (tracer).

We proceed to rewrite the TRM-G form of the mean tracer budget Eq. (4) as

$$
\bar{T}_{t}+\left(\overline{\boldsymbol{u}}-\underset{\neg}{\nabla} B_{b}\right) \cdot \nabla \bar{T}=\nabla \cdot K_{T} \nabla \bar{T}-\underset{\neg}{\nabla} \cdot \nabla \bar{T}+\bar{Q}_{T}
$$

where $B=B_{b}-B_{T}$ denotes the difference in the streamfunction for eddy-induced velocities for the mean tracer and buoyancy. By rewriting the mean tracer budget Eq. (4) as Eq. (7) we made sure that tracer and buoyancy share identical residual velocities, i.e. that on the left hand side of Eq. (7) the same eddy-induced velocity, $-\underset{\neg}{ } B_{b}$, shows up as in the mean buoyancy budget. In consequence, we only have to parameterise a single eddy-induced velocity, i.e. the one for buoyancy, for which a parameterisation similar to that of Gent and McWilliams (1990) could be used.

However, we now have to take care of the right hand side of Eq. (7). Our aim is to express it as isopycnal and diapycnal diffusion. Therefore, the mean tracer budget is written as

$$
\bar{T}_{t}+\left(\overline{\boldsymbol{u}}-\underset{\neg}{\nabla} B_{b}\right) \cdot \nabla \bar{T}=\nabla \cdot\left(\frac{K_{I}}{1+s^{2}}\left(\begin{array}{cc}
1 & -s \\
-s & s^{2}
\end{array}\right) \nabla \bar{T}\right)+\nabla \cdot\left(\frac{K_{D}}{1+s^{2}}\left(\begin{array}{cc}
s^{2} & s \\
s & 1
\end{array}\right) \nabla \bar{T}\right)+\bar{Q}_{T}(8)
$$

\footnotetext{
${ }^{1}$ The vector subscript $\neg$ shall denote anti-clockwise rotation of a two-dimensional vector by $90^{\circ}$.
} 
where $K_{I}$ denotes isopycnal diffusivity, $K_{D}$ diapycnal diffusivity and $s=\bar{b}_{y} / \bar{b}_{z}$ the negative slope of the mean isopycnals. Note that by using the slope in our formulation we have to restrict to cases with $\bar{b}_{z} \neq 0$. Now we compare the eddy flux representations on the right hand side of Eq. (7) and Eq. (8), i.e. we solve the system

$$
\left(\begin{array}{cc}
K_{T} & -B \\
B & K_{T}
\end{array}\right) \nabla T=\frac{K_{I}}{1+s^{2}}\left(\begin{array}{cc}
1 & -s \\
-s & s^{2}
\end{array}\right) \nabla T+\frac{K_{D}}{1+s^{2}}\left(\begin{array}{cc}
s^{2} & s \\
s & 1
\end{array}\right) \nabla T
$$

for $K_{I}$ and $K_{D}$. We obtain after some algebra

$$
K_{I}=K_{T}-B \frac{1+s t}{t-s}=K_{T}-\frac{B}{\tan \phi} \quad \text { and } \quad K_{D}=K_{T}+B \frac{t-s}{1+s t}=K_{T}+B \tan \phi
$$

where $t=\bar{T}_{y} / \bar{T}_{z}$ denotes the negative slope of mean tracer contours and where $\phi$ is the angle between the gradients of $\bar{T}$ and $\bar{b}$ (or the angle between isopycnals and isolines of the mean tracer). Note that there is a singularity for $t=s$ or $\phi=0$ but in that case isopycnals and tracer isolines coincide and isopycnal diffusion is then meaningless, i.e. the value of $K_{I}$ is not relevant anymore (furthermore, $B=0$ in that case, see below).

In the TRM-G framework of Eden et al. (2007a), it was shown that the diapycnal diffusivity $K_{b}$ vanishes in steady state if there is no small-scale process or interior source $Q_{b}$ acting on the buoyancy $b$. The same holds for the tracer $T$, for its interior sources $Q_{T}$ and the diffusivity $K_{T}$. On the other hand, the ocean interior is not adiabatic, there is always (weak) small-scale mixing of buoyancy and sources and sinks for $T$ might be significant. If one assumes that slopes of tracers and buoyancy are small in the ocean interior, specifically that $|s t| \ll 1$, and that $B$ is larger or at least of the same order of magnitude as $K_{T}$, the following expression will be a good approximation

$$
K_{I} \approx-\frac{B}{t-s}
$$

In other words, in the interior of the ocean, the isopycnal diffusivity is approximately given by the difference in the streamfunctions for eddy-induced velocities of tracer and buoyancy divided by the difference in their (negative) slopes.

\section{Isopycnal diffusivity in three dimensions}

We proceed with a discussion of the more relevant three-dimensional case. The zonal average from the previous section is now replaced by a mean over time (where it is assumed that the mean of all deviation vanishes) and the two-dimensional velocity vector and the $\nabla$-operator are replaced by their three-dimensional form in this section. The mean buoyancy equation and tracer equation in the 
TRM-G framework are given by

$$
\begin{aligned}
\bar{b}_{t}+\left(\overline{\boldsymbol{u}}+\nabla \times \boldsymbol{B}_{b}\right) \cdot \nabla \bar{b} & =\nabla \cdot K_{b} \nabla \bar{b}+\bar{Q}_{b} \\
\bar{T}_{t}+\left(\overline{\boldsymbol{u}}+\nabla \times \boldsymbol{B}_{T}\right) \cdot \nabla \bar{T} & =\nabla \cdot K_{T} \nabla \bar{T}+\bar{Q}_{T}
\end{aligned}
$$

Following Eden et al. (2007a), we have used the eddy flux decomposition $\overline{\boldsymbol{u}^{\prime} b^{\prime}}=-K_{b} \nabla b+\boldsymbol{B}_{b} \times \nabla b+$ $\nabla \times \boldsymbol{\theta}_{b}$ introducing the diapycnal diffusivity $K_{b}$ and the vector streamfunction for the eddy-driven advection and an equivalent flux decomposition for $\overline{\boldsymbol{u}^{\prime} T^{\prime}}$. The rotational eddy buoyancy flux is given by $\nabla \times \boldsymbol{\theta}_{b}$ using again the choice of Eden et al. (2007a) for the vector streamfunction $\boldsymbol{\theta}_{b}$ of the rotational flux. The advective part of the eddy buoyancy flux is given by the vector streamfunction $\boldsymbol{B}_{b}=-|\nabla b|^{-2}\left(\overline{\boldsymbol{u}^{\prime} b^{\prime}}-\nabla \times \boldsymbol{\theta}_{b}\right) \times \nabla b$ where we have used the gauge condition $\boldsymbol{B}_{b} \cdot \nabla b=0$. Note that an equivalent expression holds for $\boldsymbol{B}_{T}=-|\nabla T|^{-2}\left(\overline{\boldsymbol{u}^{\prime} T^{\prime}}-\nabla \times \boldsymbol{\theta}_{T}\right) \times \nabla T$ and that the diffusivities are given by $K_{b}=-|\nabla \bar{b}|^{-2}\left(\overline{\boldsymbol{u}^{\prime} b^{\prime}}-\nabla \times \boldsymbol{\theta}_{b}\right) \cdot \nabla \bar{b}$ and $K_{T}=-|\nabla \bar{T}|^{-2}\left(\overline{\boldsymbol{u}^{\prime} T^{\prime}}-\nabla \times \boldsymbol{\theta}_{T}\right) \cdot \nabla \bar{T}$. Following the two-dimensional example, we rewrite the mean tracer budget as

$$
\bar{T}_{t}+\left(\overline{\boldsymbol{u}}+\nabla \times \boldsymbol{B}_{b}\right) \cdot \nabla \bar{T}=\nabla \cdot K_{T} \nabla \bar{T}+\nabla \times \boldsymbol{B} \cdot \nabla \bar{T}+\bar{Q}_{T}
$$

with $\boldsymbol{B}=\boldsymbol{B}_{b}-\boldsymbol{B}_{T}$. As before, we aim to represent the eddy flux representation on the right hand side of Eq. (13) as isopycnal and diapycnal diffusion. The difference to the two-dimensional case, however, is that we now need two degrees of freedom for the isopycnal diffusivity, i.e. a tensor for anisotropic isopycnal diffusivity, which complicates the algebra somewhat.

There are many possibilities for an anisotropic formulation of isopycnal diffusion. Here, we will refer to isopycnal diffusion in the zonal and meridional directions. The details of the algebraic derivation and in particular our choice for the anisotropic isopycnal diffusion tensor are given in Appendix A, the result is however analogous to the two-dimensional case within the small slope approximation. We find that

$$
K_{I}^{(x)} \approx \frac{B_{2}}{t_{x}-s_{x}} \text { and } K_{I}^{(y)} \approx-\frac{B_{1}}{t_{y}-s_{y}}
$$

where $K_{I}^{(x)}$ denotes zonal isopycnal diffusivity, $K_{I}^{(y)}$ denotes meridional isopycnal diffusivity, $s_{x}, t_{x}, s_{y}$ and $t_{y}$ zonal and meridional negative slopes of isopycnals and tracer isolines respectively and where $\boldsymbol{B} \approx\left(B_{1}, B_{2}, 0\right)^{T}$. In analogy to the two-dimensional case, the zonal and meridional isopycnal diffusivities are related to the difference in the meridional and zonal component of the streamfunction for the eddy-induced velocities divided by the difference in (negative) slopes of isopycnals and tracer surfaces. 


\footnotetext{
${ }^{2}$ The numerical code together with all configurations used in this study can be accessed at http://www.ifmgeomar.de/ spflame.
}

In this section we discuss isopycnal diffusivities diagnosed from a mesoscale-eddy-permitting model of the North Atlantic Ocean with horizontal resolution of $1 / 12^{\circ} \cos \phi \times 1 / 12^{\circ}$ (where $\phi$ denotes latitude) ranging from about $10 \mathrm{~km}$ at the equator to about $5 \mathrm{~km}$ in high latitudes. The model domain extends from $20^{\circ} \mathrm{S}$ to $70^{\circ} \mathrm{N}$ with open boundaries (Stevens, 1990) at the northern and southern boundaries, with a restoring zone in the eastern Mediterranean Sea and with climatological surface forcing (Barnier et al., 1995). There are 45 vertical geopotential levels with increasing thickness with depth, ranging from $10 \mathrm{~m}$ at the surface to $250 \mathrm{~m}$ near the maximal depth of $5500 \mathrm{~m}$. The model is based on a rewritten version ${ }^{2}$ of MOM2 (Pacanowski, 1995) and is identical to the one used in e.g. Eden et al. (2007b) where more details about the model configuration can be found.

After the 10 year spin-up phase, the ocean model was integrated for additional 20 years coupled to a nitrate-based, four compartment ecosystem model which is identical to the one in Oschlies and Garçon (1998) and Eden and Oschlies (2006). Also simulated by the ocean model are dissolved oxygen and dissolved inorganic carbon (DIC). For the surface flux forcing of the latter we are using a preindustrial atmospheric partial pressure of $\mathrm{CO}_{2}$. Oxygen, DIC and nitrate are subject to sources and sinks from the remineralisation of sinking organic matter as simulate by the ecosystem model. The biological sources are linearly related since fixed Redfield ratios of organic matter was assumed. Eddy fluxes of nitrate, DIC and oxygen as given by the biogeochemical model are averaged over the last five years of the simulation from which isopycnal diffusivities are calculated according to Eq. (14). In addition, eddy fluxes of buoyancy (referenced to sea surface), temperature and salinity are averaged over the same period. Note that in order to remove the seasonal cycle, seasonal means over the five years have been averaged.

In contrast to the dynamical active tracers, DIC, oxygen and nitrate have rather large interior sources and sinks related to remineralisation of sinking organic matter. Although the biogeochemical tracers share therefore linearly dependent interior source functions their surface boundary conditions are rather different: nitrate has zero surface flux in the model, while surface fluxes of oxygen and DIC are modelled using standard bulk formulae (Wanninkhof, 1992). Note, however, that the effective restoring time scale for the surface fluxes are different for oxygen and DIC because of the large buffering effect of the oceanic carbon system. Note also that we use sea surface salinity restoring and a form of Haney restoring for temperature (Barnier et al., 1995). All forcing functions are climatological.

Fig. 1 shows the horizontal components of the streamfunction of eddy-driven advection, $\boldsymbol{B}$, for

\section{Isopycnal diffusivity in an ocean model}


buoyancy, oxygen, salinity and nitrate at $300 \mathrm{~m}$ depth. Also shown are contour lines of the respective mean tracers. Note that we have not accounted for any rotational fluxes in this analysis (see discussion at the end of section 5). Although there are similarities over certain regions, all mean tracers show in general rather different large-scale lateral and vertical structures. In consequence, the simulation yields different eddy fluxes for individual tracers and also different eddy streamfunctions. In general, largest differences between $\boldsymbol{B}$ for the individual tracers show up where gradients of the mean tracers are largest, i.e. in the tropical North Atlantic, at the southern boundary of the subtropical gyre and in particular in the western boundary current system. Note that in the subpolar North Atlantic, the results are affected by the seasonal mixed layer extending to $200 \mathrm{~m}$ depth and should be viewed therefore with caution.

Fig. 2 shows the zonal and meridional isopycnal diffusivities $K_{I}^{(x)}$ and $K_{I}^{(y)}$ at $300 \mathrm{~m}$ depth estimated from the eddy fluxes of oxygen, salinity, nitrate and DIC. It is evident that the results for the individual tracers are very similar. The same holds for temperature (not shown) although here the difference between the slopes for temperature and buoyancy often becomes very small such that isopycnal diffusivity is not meaningful anymore. Accordingly, the spatial correlations between $K_{I}^{(x)}$ and $K_{I}^{(y)}$ estimated from the different tracers are rather high at $300 \mathrm{~m}$ and range between 0.4 and 0.5 (Table 1) except for correlations with salinity which become lower for certain combinations with the other tracers which we might also relate to the small differences in slopes of isopycnals and isohalines. Considering the depth range $200 m$ to $2500 m$ (Table 1 ) the spatial correlations decrease little and are still high. Over large regions zonal and meridional diffusivities are positive with rather large lateral inhomogeneities with values ranging between 0 and $5000 \mathrm{~m}^{2} / \mathrm{s}$, but there are also regions with negative diffusivities, i.e. near the Azores Front for $K_{I}^{(y)}$ and the north-western flank of the North Atlantic Current for $K_{I}^{(x)}$. It is also evident that $K_{I}^{(x)}$ is in general larger than $K_{I}^{(y)}$. In fact, in the tropical Atlantic $K_{I}^{(y)}$ is almost vanishing for all tracers, while $K_{I}^{(x)}$ is large with maxima below the Equatorial Undercurrent and the North Equatorial Counter Current.

Fig. 3 shows the results for salinity and oxygen at $1200 \mathrm{~m}$ in the subtropical gyre. Here, a particularly large difference in the mean tracer gradients shows up: While the isolines of the mean oxygen are tilted roughly along the north-east/south-west direction, the mean salinity shows the familiar maximum near the Mediterranean outflow region offshore of the Strait of Gibraltar in this depth range as the most prominent feature. The effect is that lateral gradients of salinity and oxygen are becoming almost perpendicular over large regions of the subtropical North Atlantic. Nevertheless, $K_{I}^{(x)}$ and $K_{I}^{(y)}$ diagnosed from both tracers are very similar. The figure shows also that the anisotropy seen already in Fig. 2 with larger $K_{I}^{(x)}$ (at $1200 \mathrm{~m}$ depth around $1000 \mathrm{~m}^{2} / \mathrm{s}$ ) and much smaller $K_{I}^{(y)}$ (at $1200 \mathrm{~m}$ depth almost vanishing) over wide regions of the subtropical gyre, extends also to the 
deeper levels.

In general, both $K_{I}^{(x)}$ and $K_{I}^{(y)}$ decrease with depth. Since the results from each individual tracer are very similar we show in Figure 4 the average over three estimates (DIC, oxygen and nitrate) at sections at $30^{\circ} \mathrm{W}$ and $30^{\circ} \mathrm{N}$. In general, isopycnal diffusivities are large in the main thermocline and above and decay to almost zero below, which is similar to a previous estimate of the thickness diffusivity (Eden et al., 2007b). It is again obvious that meridional diffusivities are much smaller than zonal isopycnal diffusivities. Very similar results are obtained using temperature and salinity.

\section{Discussion and conclusions}

In this study we have diagnosed isopycnal diffusivities from the simulation of five independent tracer simulations of a realistic mesoscale-eddy-permitting model of the North Atlantic coupled to a biogeochemical model. Using the TRM-G framework of Eden et al. (2007a) and assuming identical eddy-driven advection velocities for buoyancy and tracer, we found that in the zonal mean case the isopycnal diffusivity is simply given by the difference in the streamfunctions for eddy-driven advection of buoyancy and the respective tracer, divided by the difference in the negative slopes of buoyancy and tracer. While for the two-dimensionally zonal mean case a scalar isopycnal diffusivity is sufficient, for the three-dimensional case of temporal averaging an isopycnal diffusivity tensor with two independent components is needed to describe the mesoscale eddy effects, in analogy to what have been found by Eden et al. (2007b) for the thickness diffusivity appropriate to the Gent and McWilliams (1990) parameterisation.

Although other possibilities to define such anisotropic isopycnal diffusivities are certainly possible, we have diagnosed the isopycnal diffusivity from the eddying model in terms of a zonal $\left(K_{I}^{(x)}\right)$ and meridional $\left(K_{I}^{(y)}\right)$ isopycnal diffusivity. The diagnosis shows similar results independent of the tracer under investigation, even when the lateral and vertical gradients of different tracers are almost perpendicular to each other. Our results therefore support the use of a single eddy-advection velocity and a single isopycnal diffusivity for all tracers in ocean models. In fact, we have not expected such a good agreement for the different isopycnal diffusivities of different tracers, since all tracers have different mean distributions and rather different interior forcing and surface forcing. One reason for the good agreement might be the fact that all tracers have weak diabatic forcing (sources and sinks), i.e. have a high Peclet number with respect to meso-scale flow.

The results also support to use identical thickness and isopycnal diffusivities. Fig. 5 shows the zonal $\left(K^{(x)}\right)$ and meridional thickness diffusivity $\left(K^{(y)}\right)$ appropriate to the Gent and McWilliams 
(1990) parameterisation at $300 \mathrm{~m}$ which are given by the relation

$$
\overline{\boldsymbol{u}_{h}^{\prime} b^{\prime}}=\left(\begin{array}{cc}
K^{(x)} & 0 \\
0 & K^{(y)}
\end{array}\right) \nabla_{h} \bar{b}
$$

where $\boldsymbol{u}_{h}^{\prime}$ denotes the horizontal velocity fluctuations and $\nabla_{h}$ the horizontal part of $\nabla$ (see also Eden et al. (2007b) for the definition of anisotropic thickness diffusivity). As for the diagnosis of isopycnal diffusivities $\left(K_{I}^{(x)}\right.$ and $K_{I}^{(y)}$ ), no attempt was made to remove rotational eddy fluxes for estimating the thickness diffusivities $K^{(x)}$ and $K^{(y)}$ (see discussion below). Fig. 5 shows indeed that the magnitude and the lateral (and vertical, not shown) structure of $K^{(x)}$ and $K^{(y)}$ is similar to our estimates of isopycnal diffusivity $K_{I}^{(x)}$ and $K_{I}^{(y)}$. Spatial correlations between isopyncal and thickness diffusivities at $300 \mathrm{~m}$ depth and also for the depth range of the thermocline (Table 2) show similar values as the correlations of isopycnal diffusivities amongst themself (Table 1).

On the other hand, the diagnosis also showed the need of an anisotropic isopycnal diffusivity operator as found before for the thickness diffusivity (Eden et al., 2007b). Zonal isopycnal diffusivity is in general larger than meridional diffusivity. This anisotropy is in particular large in the tropical Atlantic, where the meridional diffusivity almost vanishes. A possible explanation might be different regimes in geophysical turbulence due to an equatorward energy cascade as suggested by Theiss (2004), i.e. isotropic turbulence in higher latitudes and anisotropic turbulence in low latitudes, for which the latter is influenced by zonal energy radiation by Rossby waves as anticipated by Rhines (1975). The transition between both regimes was found by Eden (2007) to be roughly located at $30^{\circ} \mathrm{N}$, which was recently supported by Tulloch et al. (2008).

There is also a strong depth dependency in the isopycnal diffusivities as already noted by Eden et al. (2007b) and Eden (2006) for the thickness diffusivity. A similar decay with depth was also found by Ferreira et al. (2005) with an inverse modeling approach. A concise explanation for this prominent vertical structure is presently lacking, but we note here that the recently proposed closure for the thickness diffusivity of Eden and Greatbatch (2008) based on Green's (1970) mixing length assumption for the diffusivity, yields a similar depth dependency as diagnosed here for the isopycnal diffusivity.

The effect of strong anisotropic isopycnal diffusivity on the ventilation of the interior of the ocean is in particular relevant for estimates of the oceanic carbon uptake. In the present study, we can only speculate about the effect and leave the detailed discussion for future studies. However, it is clear that the low meridional isopycnal diffusivity might prevent a significant meridional diffusive transport of DIC into the thermocline, leaving advection as the main subduction mechanism in the meridional direction. We also note that the ventilation of the shadow zones in the mid-depth tropical ocean, 
where lowest oxygen concentrations are found and which are thought to be important for the global nutrient cycling, is strongly controlled by isopycnal (and diapycnal) mixing. Therefore, anisotropic isopycnal mixing might also have a strong effect on the volume and extent and the future fate of the oxygen minimum zones.

We have not accounted for rotational fluxes in the present analysis. Eden et al. (2007b) found improvements in the structure of the diagnosed thickness diffusivities, by carefully removing physically meaningful rotational fluxes following Marshall and Shutts (1981) and Eden et al. (2007a). These improvements are given by a reduction of regions of negative thickness diffusivities in the diagnosis. However, here we found that by using identical definitions for rotational fluxes as in Eden et al. (2007a), the magnitudes of the diagnosed isopycnal diffusivities become very large with fluctuating signs. Although the energetic constraint on spatially varying, zonal and meridional isopycnal diffusivities are more complex than for a constant isotropic diffusivity (which should be positive to insure globally variance dissipation), diffusivities of large magnitude with fluctuating signs appear physically unreasonable to us. We therefore conclude that a removal of rotational fluxes following Eden et al. (2007a) does not yield an improved estimate of isopycnal diffusivities in this case. We speculate that the reason for this failure might be the fact that the definition for isopycnal diffusivities is given by differences (both in eddy streamfunctions and slopes), while the thickness diffusivity is estimated from the fluxes themselves. Therefore, small errors in the calculation of the rotational fluxes might affect the results stronger for isopycnal diffusivities and less for the diagnosis of thickness diffusivities.

\section{Acknowledgements}

This study was supported by the German DFG as part of the SFB 754. The model integrations have been performed on a NEX-SX8 at the University Kiel and on a NEC-SX6 at the Deutsches Klimarechenzentrum (DKRZ), Hamburg.

\section{Appendix A}

In this appendix we detail our choice and derivation of the anisotropic isopycnal diffusion tensor and its relation to the TRM-G framework. There are many possibilities for an anisotropic formulation of isopycnal diffusion. Here, we will refer to isopycnal diffusion in zonal and meridional direction. For simplicity, we first review the derivation of the diapycnal diffusivity and follow this example to derive isopycnal diffusivities in the zonal and meridional directions. We start be defining a unit vector pointing along the buoyancy gradient $\boldsymbol{n}_{b}=\nabla \bar{b} /|\nabla \bar{b}|$ and note that the diapycnal component of the 
eddy tracer flux, $\boldsymbol{F}=\overline{\boldsymbol{u}^{\prime} T^{\prime}}$, can be expressed as $\left(\boldsymbol{F} \cdot \boldsymbol{n}_{b}\right) \boldsymbol{n}_{b}=\left(\boldsymbol{n}_{b} \boldsymbol{n}_{b}\right) \cdot \boldsymbol{F}$, which defines the $(3 \times 3)$ tensor $\boldsymbol{n}_{b} \boldsymbol{n}_{b}$ given by

$$
\boldsymbol{n}_{b} \boldsymbol{n}_{b}=\frac{1}{1+s_{x}^{2}+s_{y}^{2}}\left(\begin{array}{ccc}
s_{x}^{2} & s_{y} s_{x} & s_{x} \\
s_{x} s_{y} & s_{y}^{2} & s_{y} \\
s_{x} & s_{y} & 1
\end{array}\right)
$$

with the zonal and meridional negative slopes of the mean isopycnals $s_{x}=\bar{b}_{x} / \bar{b}_{z}$ and $s_{y}=\bar{b}_{y} / \bar{b}_{z}$. The vector $\left(\boldsymbol{F} \cdot \boldsymbol{n}_{b}\right) \boldsymbol{n}_{b}$ can now be expressed as a down-gradient flux of the mean tracer $T$

$$
\boldsymbol{F}_{d i a}=\left(\boldsymbol{F} \cdot \boldsymbol{n}_{b}\right) \boldsymbol{n}_{b}=K_{D}\left(\boldsymbol{n}_{b} \boldsymbol{n}_{b}\right) \cdot \nabla T
$$

where $K_{D}$ can always be chosen appropriately as long as $\nabla T$ is not perpendicular to $\boldsymbol{n}_{b}$. The flux $\boldsymbol{F}_{d i a}$ is a diapycnal diffusive flux and its divergence resembles diapycnal diffusion with the diapycnal diffusivity $K_{D}$. We now specify two additional vectors pointing along the isopycnal direction and in zonal and meridional direction

$$
\boldsymbol{n}_{1}=\boldsymbol{e}_{2} \times \boldsymbol{n}_{b}=\frac{1}{|\nabla b|}\left(\begin{array}{c}
b_{z} \\
0 \\
-b_{x}
\end{array}\right) \quad \text { and } \quad \boldsymbol{n}_{2}=\boldsymbol{e}_{1} \times \boldsymbol{n}_{b}=\frac{1}{|\nabla b|}\left(\begin{array}{c}
0 \\
-b_{z} \\
b_{y}
\end{array}\right)
$$

where $\boldsymbol{e}_{1}$ and $\boldsymbol{e}_{2}$ are unit vectors in the zonal and meridional directions respectively. Note that for sloping isopycnals, the magnitude of $\boldsymbol{n}_{1}$ and $\boldsymbol{n}_{2}$ might differ from one, i.e. they are not unit vectors, but we ignore this issue here for simplicity, since the deviation is small for small slopes, an assumption we will employ below anyway. The corresponding parameterised components of the eddy tracer flux $\boldsymbol{F}$ are given by

$$
\boldsymbol{F}_{i s o}^{(x)}=K_{I}^{(x)}\left(\boldsymbol{n}_{1} \boldsymbol{n}_{1}\right) \cdot \nabla T \quad \text { and } \quad \boldsymbol{F}_{i s o}^{(y)}=K_{I}^{(y)}\left(\boldsymbol{n}_{2} \boldsymbol{n}_{2}\right) \cdot \nabla T
$$

where $K_{I}^{(x)}$ and $K_{I}^{(y)}$ resemble isopycnal, zonal and meridional diffusivities which can always be chosen appropriately as for $K_{D}$. Taking both tensors together and using $s_{x}^{2}, s_{y}^{2} \ll 1$ as above we obtain

$$
\boldsymbol{K}_{i s o}=\left(\begin{array}{ccc}
K_{I}^{(x)} & 0 & -K_{I}^{(x)} s_{x} \\
0 & K_{I}^{(y)} & -K_{I}^{(y)} s_{y} \\
-K_{I}^{(x)} s_{x} & -K_{I}^{(y)} s_{y} & K_{I}^{(x)} s_{x}^{2}+K_{I}^{(y)} s_{y}^{2}
\end{array}\right)
$$

with $s=\left(s_{x}, s_{y}\right)^{T}$. Note that for $K_{I}^{(x)}=K_{I}^{(y)}$ we obtain the standard form of the isopycnal diffusivity tensor within the small-slope approximation (Gent and McWilliams, 1990). In analogy to the two-dimensional case, we proceed by comparing the eddy flux representation on the right hand side of Eq. (13) with the mean tracer budget expressed using the isopycnal and diapycnal diffusivity tensor, i.e. solving the system

$$
\left(\begin{array}{ccc}
K_{T} & -B_{3} & B_{2} \\
B_{3} & K_{T} & -B_{1} \\
-B_{2} & B_{1} & K_{T}
\end{array}\right) \nabla T=\left(\begin{array}{ccc}
K_{I}^{(x)}+K_{D} s_{x}^{2} & K_{D} s_{x} s_{y} & \left(K_{D}-K_{I}^{(x)}\right) s_{x} \\
K_{D} s_{x} s_{y} & K_{I}^{(y)}+K_{D} s_{y}^{2} & \left(K_{D}-K_{I}^{(y)}\right) s_{y} \\
\left(K_{D}-K_{I}^{(x)}\right) s_{x} & \left(K_{D}-K_{I}^{(y)}\right) s_{y} & K_{I}^{(x)} s_{x}^{2}+K_{I}^{(y)} s_{y}^{2}+K_{D}
\end{array}\right) \nabla \bar{T}(21)
$$


for $K_{I}^{(x)}, K_{I}^{(y)}$ and $K_{D}$, where $B_{1}, B_{2}$ and $B_{3}$ denote the components of the streamfunction $\boldsymbol{B}$ with $\left(B_{1}, B_{2}, B_{3}\right)^{T}=\boldsymbol{B}$. Using again $s_{x}^{2}, s_{y}^{2} \ll 1$ we find

$$
\begin{aligned}
K_{I}^{(x)}\left(t_{x}-s_{x}\right) & =K_{T}\left(t_{x}-s_{x}\right)-B_{3} t_{y}+B_{2} \\
K_{I}^{(y)}\left(t_{y}-s_{y}\right) & =K_{T}\left(t_{y}-s_{y}\right)+B_{3} t_{x}-B_{1}
\end{aligned}
$$

introducing the negative slopes of the mean tracer $t_{y}=\bar{T}_{y} / \bar{T}_{z}$ and $t_{x}=\bar{T}_{x} / \bar{T}_{z}$ in meridional and zonal direction, respectively. Since $\left|B_{3}\right| \ll\left|B_{1}\right|,\left|B_{2}\right|$ when the slopes are small (because of the condition $\left.\boldsymbol{B}_{b} \cdot \nabla \bar{b}=0\right)$ and assuming that the order of magnitude $K_{T}$ is at least less or equal than the magnitudes of $B_{1}$ and $B_{2}$ we find that

$$
K_{I}^{(x)} \approx \frac{B_{2}}{t_{x}-s_{x}} \quad \text { and } \quad K_{I}^{(y)} \approx-\frac{B_{1}}{t_{y}-s_{y}}
$$

For the diapycnal diffusivity we find neglecting again terms $o\left(s^{2}\right)$ that

$$
K_{D}=K_{T}+B_{1}\left(t_{y}-s_{y}\right)-B_{2}\left(t_{x}-s_{x}\right)
$$

\section{References}

Andrews, D. G., J. R. Holton, and C. B. Leovy, 1987: Middle Atmosphere Dynamics. Academic Press.

Barnier, B., L. Siefridt, and P. Marchesiello, 1995: Thermal forcing for a global ocean circulation model using a three year climatology of ECMWF analysis. J. Mar. Sys., 6, 363-380.

Bryan, K., J. K. Dukowicz, and R. D. Smith, 1999: On the mixing coefficient in the parameterization of bolus velocity. J. Phys. Oceanogr., 29, 2442-2456.

Drijfhout, S. S. and W. Hazeleger, 2001: Eddy mixing of potential vorticity versus thickness in an isopycnic ocean model. J. Phys. Oceanogr., 31(2), 481-505.

Eden, C., 2006: Thickness diffusivity in the Antarctic Circumpolar Current. Geophys. Res. Letters, 33(L11606). Doi:10.1029/2006GL026157.

Eden, C., 2007: Eddy length scales in the North Atlantic. J. Geophys. Res., 112(C06004). Doi:10.1029/2006JC003901.

Eden, C. and R. J. Greatbatch, 2008: Towards a mesoscale eddy closure. Ocean Modelling, 20, 223-239.

Eden, C., R. J. Greatbatch, and D. Olbers, 2007a: Interpreting eddy fluxes. J. Phys. Oceanogr., 37, 1282-1296.

Eden, C., R. J. Greatbatch, and J. Willebrand, 2007b: A diagnosis of thickness fluxes in an eddy-resolving model. J. Phys. Oceanogr., 37, 727-742.

Eden, C. and C. Oschlies, 2006: Adiabatic reduction of circulation-related $\mathrm{CO}_{2}$ air-sea flux biases in North Atlantic carbon-cycle models. Glob. Biochem. Cycles, 20(GV2008). Doi:10.1029/2005GB002521.

Ferreira, D., J. Marshall, and P. Heimbach, 2005: Estimating eddy stresses by fitting dynamics to observations using a residual-mean ocean circulation model and its adjoint. J. Phys. Oceanogr., 35, 1891-1910. 
Gent, P. R. and J. C. McWilliams, 1990: Isopycnal mixing in ocean circulation models. J. Phys. Oceanogr., 20, $150-155$.

Greatbatch, R. J., 2001: A framework for mesoscale eddy parameterization based on density-weighted averaging at fixed height. J. Phys. Oceanogr., 31(9), 2797-2806.

Green, J. S., 1970: Transfer properties of the large-scale eddies and the general circulation of the atmosphere. Quart. J. Royal Met. Soc., 96, 157-185.

Houghton, J. T., Y. Ding, D. J. Griggs, M. Noguer, P. J. van der Lindeni, X. Dai, K. Maskell, and C. A. Johnson, eds., 2001: Climate Change (2001) The scientific basis., chapter Contribution of Working Group 1 to the Third Assessment Report of the Intergovernmental Panel on Climate Change., p. 881. Cambridge University Press.

Jochum, M., 1997: Eine ortsabhängige Parameterisierung von Wirbelflüssen. Master's thesis, Institut für Meereskunde, Kiel.

Luyten, J. R., J. Pedlosky, and H. Stommel, 1983: The ventilated thermocline. J. Phys. Oceanogr., 23(2), 292-309.

Marshall, J. and G. Shutts, 1981: A note on rotational and divergent eddy fluxes. J. Phys. Oceanogr., 11(12), $1677-1679$.

Nakamura, M. and Y. Chao, 2000: On the eddy isopycnal thickness diffusivity of the Gent-McWilliams subgrid mixing parameterization. J. Climate, 13, 502-510.

Oschlies, A. and V. Garçon, 1998: Eddy induced enhancement of primary production in a model of the North Atlantic Ocean. Nature, 394, 266-269.

Pacanowski, R. C., 1995: MOM 2 Documentation, User's Guide and Reference Manual. Technical report, GFDL Ocean Group, GFDL, Princeton, USA.

Peterson, K. A. and R. J. Greatbatch, 2001: Vorticity fluxes in shallow water ocean models. Atmos.-Ocean., 39(1), 1-14.

Redi, M. H., 1982: Oceanic isopycnal mixing by coordinate rotation. J. Phys. Oceanogr., 12, 1154-1158.

Rhines, P., 1975: Waves and turbulence on a beta-plane. J. Fluid Mech., 69, 417-443.

Rix, N. and J. Willebrand, 1996: A note on the parameterisation of eddy-induced mixing from eddy-resolving model data. J. Phys. Oceanogr., 26, 2281-2285.

Roberts, M. J. and D. P. Marshall, 2000: On the validity of downgradient eddy closures in ocean models. $J$. Geophys. Res., 105(C12), 28.613-28.627.

Solovev, M., P. Stone, and P. Malanotte-Rizzoli, 2002: Assessment of mesoscale eddy parameterizations for a single-basin coarse-resolution ocean model. J. Geophys. Res., 107(C9), 9-1 -9-13.

Stevens, D. P., 1990: On open boundary conditions for three dimensional primitiv equation ocean circulation models. Geophys. Astrophys. Fluid Dyn., 51, 103-133.

Theiss, J., 2004: Equatorward energy cascade, critical latitude, and the predominance of cyclonic vortices in geostrophic turbulence. J. Phys. Oceanogr., 34, 1663-1678.

Treguier, A. M., 1999: Evaluating eddy mixing coefficients from eddy-resolving ocean models: A case study. $J$. Mar. Res., 57, 89-108. 
Tulloch, R., J. Marshall, and K. Smith, 2008: Interpretation of the propagation of surface altimetric observations in terms of planetary waves and geostrophic turbulence. J. Geophys. Res.. Submitted.

Wanninkhof, R., 1992: Relationship between gas exchange and wind speed over the ocean. J. Geophys. Res., 97, 7373-7381. 


\begin{tabular}{c|c|c|c|c}
$\left.K_{I}^{(x)}\right|^{300 m}$ & $O_{2}$ & $S$ & $N O_{3}$ & $D I C$ \\
\hline$O_{2}$ & 1 & 0.24 & 0.53 & 0.49 \\
\hline$S$ & 0.24 & 1 & 0.49 & 0.43 \\
\hline$N O_{3}$ & 0.53 & 0.49 & 1 & 0.53 \\
\hline$D I C$ & 0.49 & 0.43 & 0.53 & 1 \\
\hline
\end{tabular}

\begin{tabular}{c|c|c|c|c}
$\left.K_{I}^{(y)}\right|^{300 m}$ & $O_{2}$ & $S$ & $N O_{3}$ & $D I C$ \\
\hline$O_{2}$ & 1 & 0.18 & 0.43 & 0.46 \\
\hline$S$ & 0.18 & 1 & 0.09 & 0.21 \\
\hline $\mathrm{NO}_{3}$ & 0.43 & 0.09 & 1 & 0.47 \\
\hline $\mathrm{DIC}$ & 0.46 & 0.21 & 0.47 & 1 \\
\hline
\end{tabular}

\begin{tabular}{c|c|c|c|c}
$\left.K_{I}^{(x)}\right|_{2500 m} ^{200 m}$ & $O_{2}$ & $S$ & $N O_{3}$ & $D I C$ \\
\hline$O_{2}$ & 1 & 0.30 & 0.42 & 0.41 \\
\hline$S$ & 0.30 & 1 & 0.37 & 0.37 \\
\hline$N O_{3}$ & 0.42 & 0.37 & 1 & 0.47 \\
\hline$D I C$ & 0.41 & 0.37 & 0.47 & 1 \\
\hline
\end{tabular}

\begin{tabular}{c|c|c|c|c}
$\left.K_{I}^{(y)}\right|_{2500 m} ^{200 m}$ & $O_{2}$ & $S$ & $N O_{3}$ & $D I C$ \\
\hline$O_{2}$ & 1 & 0.21 & 0.37 & 0.36 \\
\hline$S$ & 0.21 & 1 & 0.20 & 0.21 \\
\hline$N O_{3}$ & 0.37 & 0.20 & 1 & 0.36 \\
\hline$D I C$ & 0.36 & 0.21 & 0.36 & 1 \\
\hline
\end{tabular}

Table 1: Spatial correlations of $K_{I}^{(x)}$ (left tables) and $K_{I}^{(y)}$ (right tables) estimated from different tracers for the horizontal domain shown in Fig. 2 and at $300 \mathrm{~m}$ depth (upper tables) and for the vertical range $200 \mathrm{~m}$ to $2500 \mathrm{~m}$ (lower tables). Regions in which the difference in the slopes of mean buoyancy and tracer are less than $10^{-6}$ and where diffusivities exceed $\pm 5000 \mathrm{~m}^{2} / \mathrm{s}$ are not used for calculating the correlation.

\begin{tabular}{l|c|c|c|c} 
& $\mathrm{O}_{2}$ & $\mathrm{~S}$ & $\mathrm{NO}_{3}$ & $\mathrm{DIC}$ \\
\hline$K_{I}^{(x)}$ vs. $\left.K^{(x)}\right|^{300 m}$ & 0.41 & 0.54 & 0.56 & 0.50 \\
\hline$K_{I}^{(y)}$ vs. $\left.K^{(y)}\right|^{300 m}$ & 0.27 & 0.29 & 0.23 & 0.32 \\
\hline$K_{I}^{(x)}$ vs. $\left.K^{(x)}\right|_{2500 m} ^{200 m}$ & 0.29 & 0.34 & 0.31 & 0.35 \\
\hline$K_{I}^{(y)}$ vs. $\left.K^{(y)}\right|_{2500 m} ^{200 m}$ & 0.19 & 0.13 & 0.20 & 0.21 \\
\hline
\end{tabular}

Table 2: Spatial correlations of zonal $\left(K_{I}^{(x)}\right)$ and meridional $\left(K_{I}^{(y)}\right)$ isopycnal diffusivity with zonal $\left(K^{(x)}\right)$ and meridional $\left(K^{(y)}\right)$ thickness diffusivity estimated from the different tracer at $300 \mathrm{~m}$ depth (first two rows) and for the depth range $200 \mathrm{~m}$ to $2500 \mathrm{~m}$ (lower two rows). Regions in which the difference in the slopes of mean buoyancy and tracer are less than $10^{-6}$ and where diffusivities exceed $\pm 5000 \mathrm{~m}^{2} / \mathrm{s}$ are not used for calculating the correlation. 

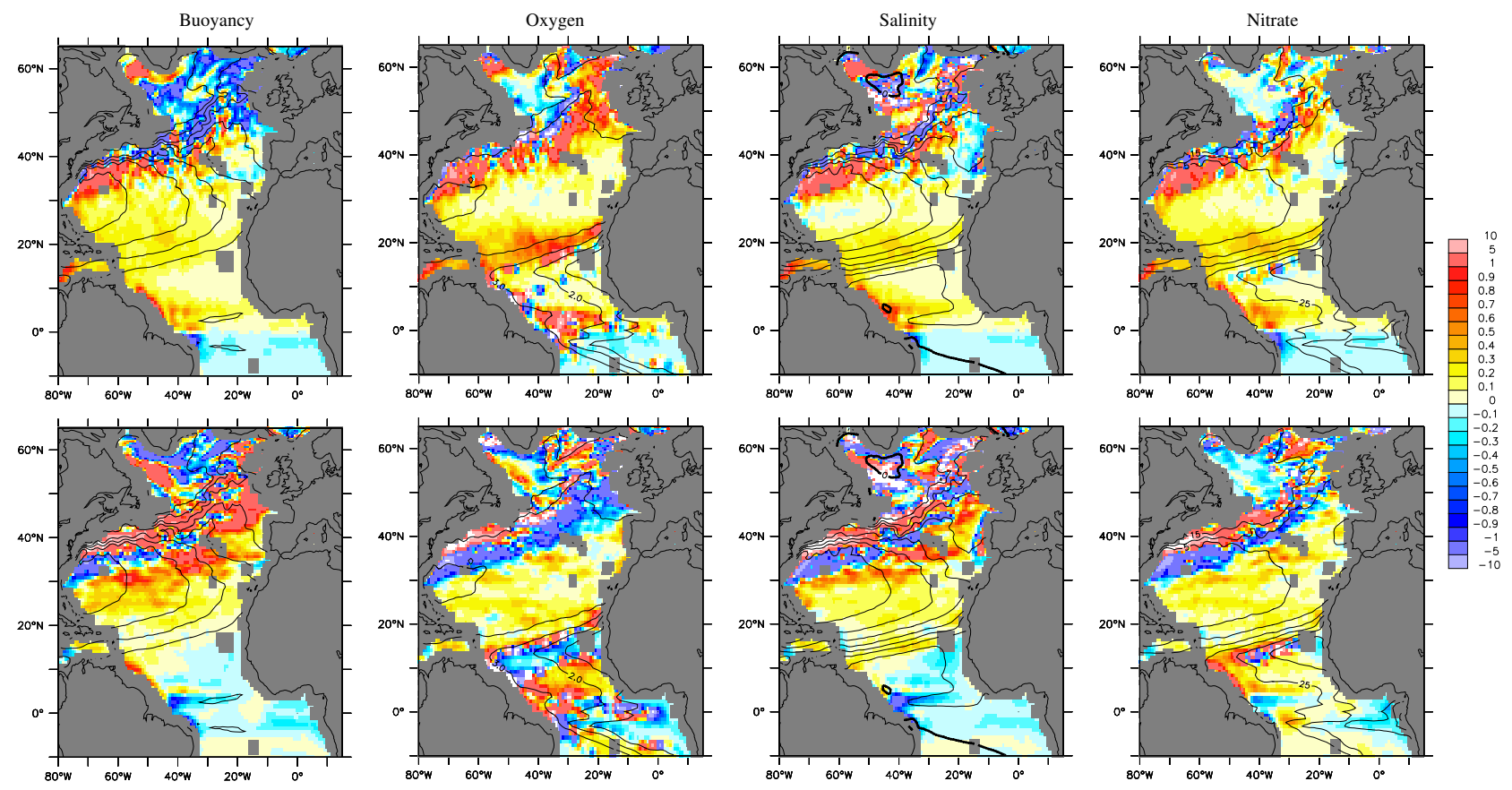

Figure 1: Upper row: Zonal component $\left(B_{1}\right)$ of the streamfunction of eddy driven advection $\left(\boldsymbol{u}^{*}=\right.$ $\nabla \times \boldsymbol{B})$ for buoyancy, oxygen, salinity and nitrate at $300 \mathrm{~m}$ depth in $\mathrm{m}^{2} \mathrm{~s}^{-1}$. Also shown are contour lines of mean tracers at $300 \mathrm{~m}$ depth. Lower row: same but for meridional component $\left(B_{2}\right)$. 

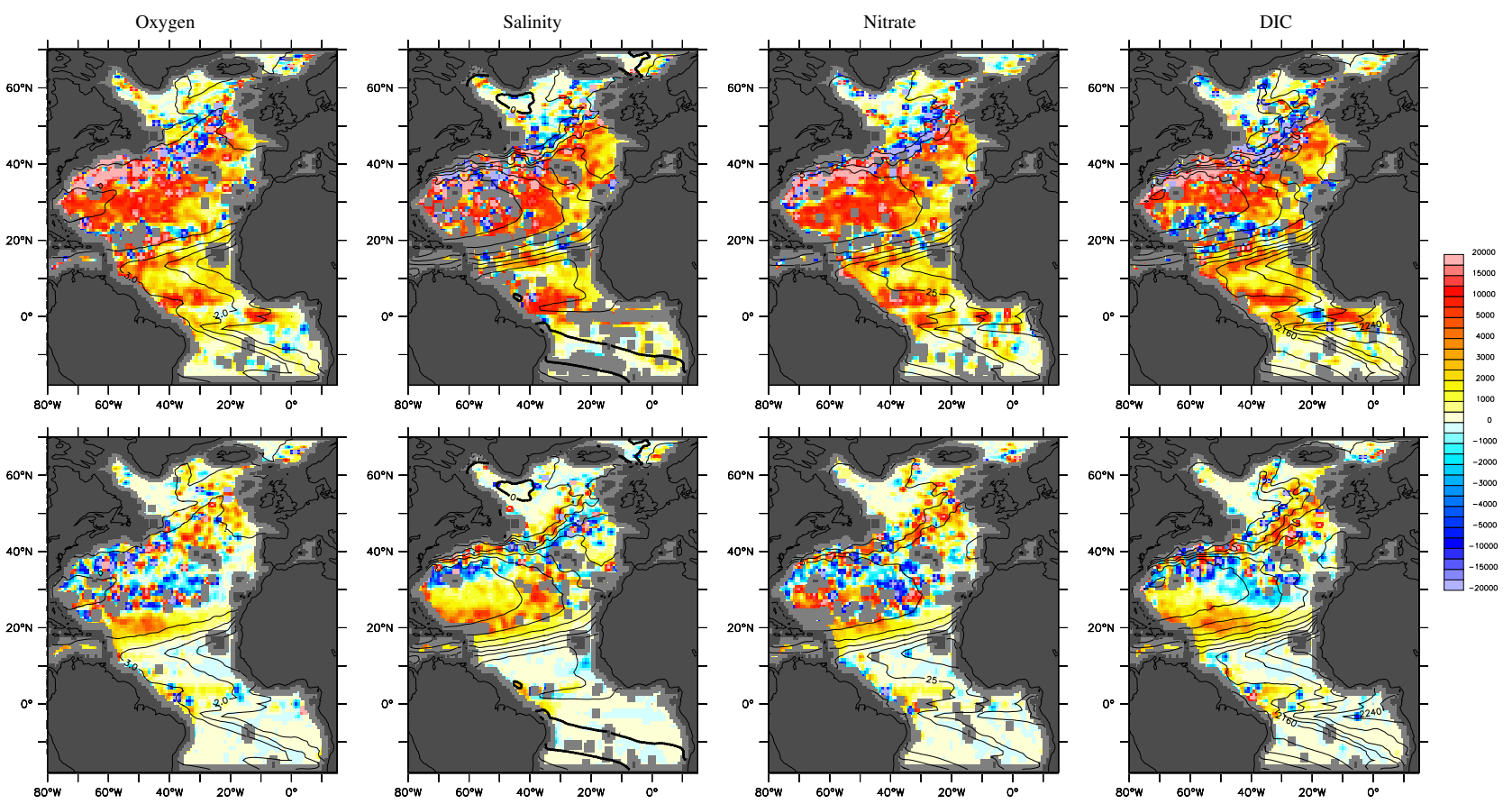

Figure 2: Upper row: Zonal isopycnal diffusivity $K_{I}^{(x)}$ in $\mathrm{m}^{2} / \mathrm{s}$ at $300 \mathrm{~m}$ depth estimated from oxygen, salinity, nitrate and DIC. Lower row: same but for $K_{I}^{(y)}$. Also shown are contours of mean tracers at $300 \mathrm{~m}$ depth. Regions in which the difference in the slopes of mean buoyancy and tracer are less than $10^{-6}$ are shaded grey.
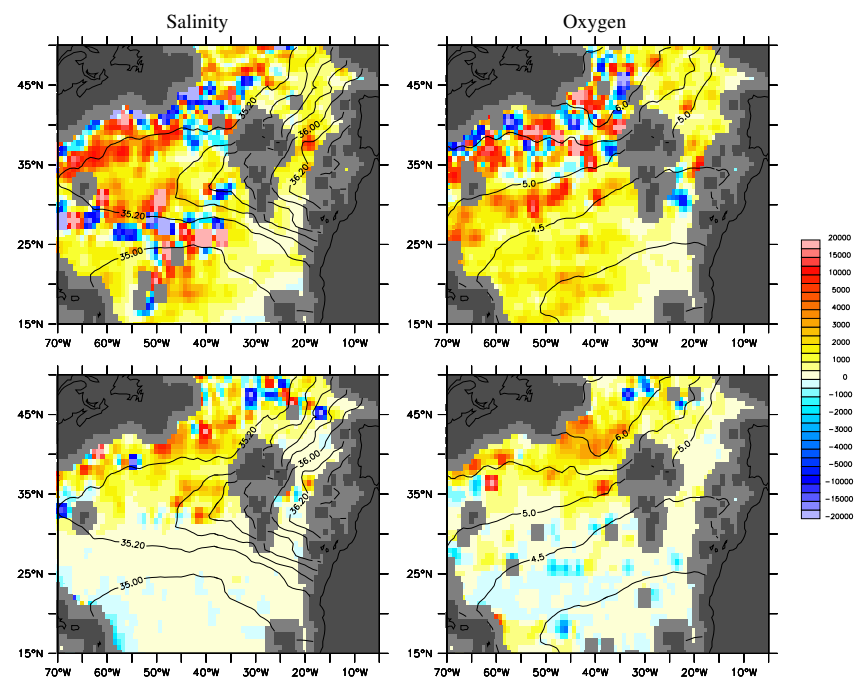

Figure 3: Same as Fig. 2 but at $1200 m$ depth and for salinity and oxygen only. 

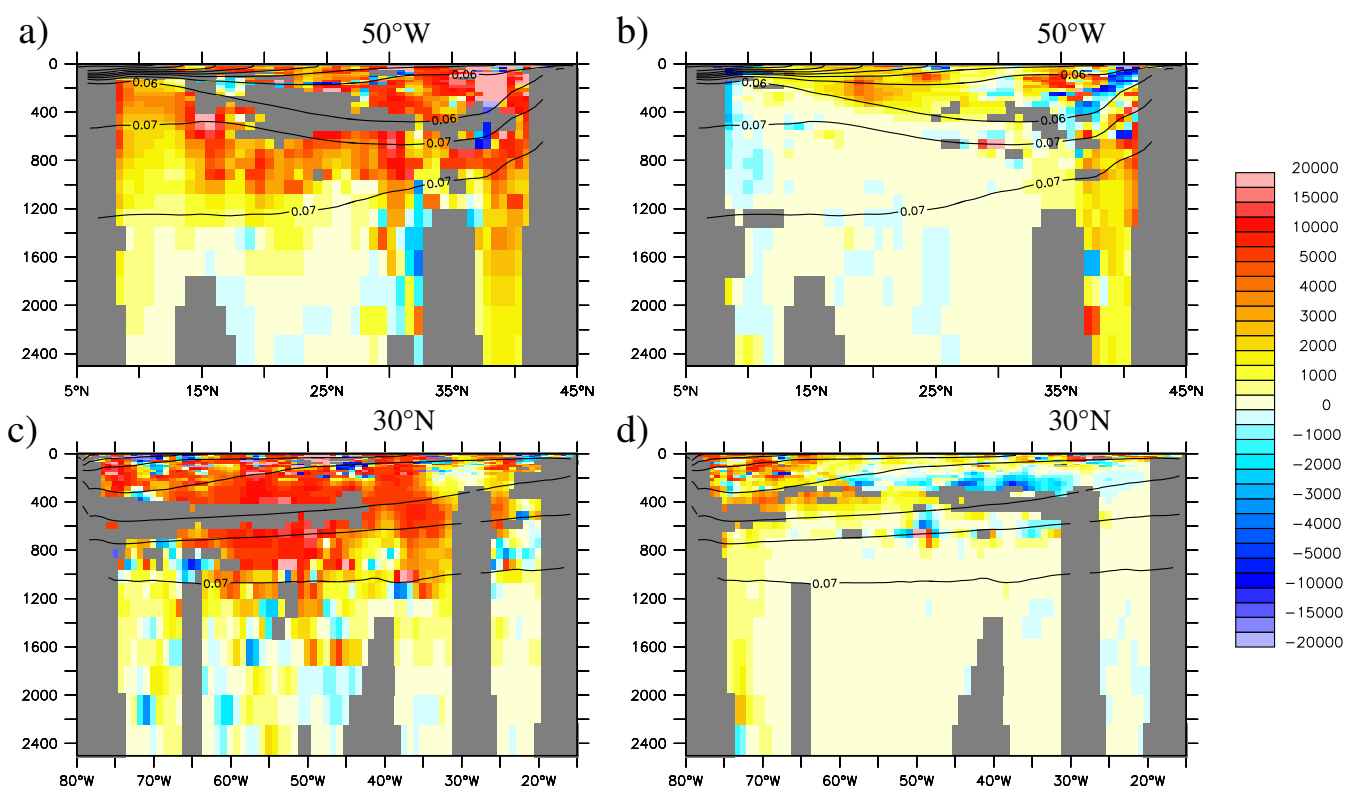

Figure 4: a) Zonal (a,c) and meridional (b,d) isopycnal diffusivity $K_{I}^{(x)}$ in $\mathrm{m}^{2} / \mathrm{s}$ at $50^{\circ} \mathrm{W}$ (a,b) and $30^{\circ} \mathrm{N}(\mathrm{c}, \mathrm{d})$. Also shown are contours of mean buoyancy.

a)

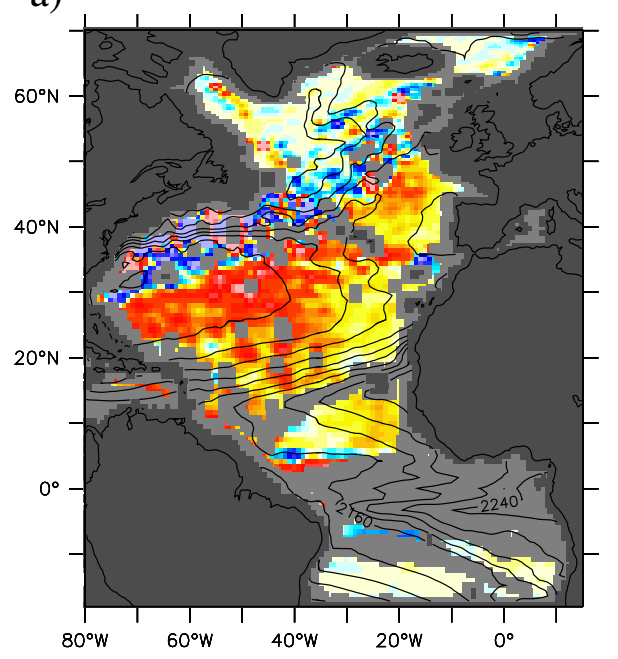

b)

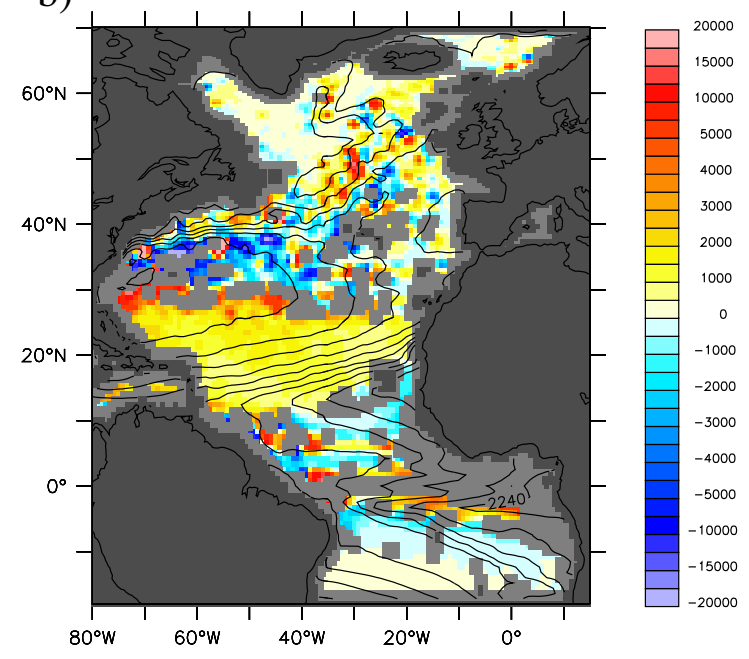

Figure 5: a) Zonal thickness diffusivity $\left(K^{(x)}\right)$ at $300 m$ depth in $m^{2} / s$. b) Same as a) but meridional thickness diffusivity $\left(K^{(y)}\right)$ Regions in which the isopycnal slopes are less than $10^{-5}$ are shaded grey. Also shown are contours of mean buoyancy at $300 m$ depth. 